\title{
PLD-fabricated isotropic Pr-Fe-B film magnets deposited on glass substrates
}

\author{
A. Yamashita ${ }^{1}$, K. Hirotaki ${ }^{1}$, A. Kurosaki ${ }^{1}$, T. Yanai $^{1}$, H. Fukunaga ${ }^{1}$, R. Fujiwara ${ }^{2}$, T. Shinshi ${ }^{2}$ and M. Nakano ${ }^{1}$ \\ ${ }^{1}$ Graduate School of Engineering, Nagasaki University, Nagasaki, Nagasaki 852-8521, Japan \\ ${ }^{2}$ Interdisciplinary Graduate School of Science and Engineering, Tokyo Institute of Technology, Suzukakedai, \\ Kanagawa 226-8503, Japan
}

\begin{abstract}
Pr-Fe-B thick-film magnets were deposited on glass substrates without a buffer layer using a PLD (Pulsed Laser Deposition) method to apply the films to MEMS (Micro-Electro-Mechanical-Systems). Large particles with the average diameter of approximately $50 \mu m$ were emitted from a target and enabled us to obtain the relatively high deposition rate of $30 \mu \mathrm{m} / \mathrm{h}$. An approximately $100 \mu \mathrm{m}$-thick PrFe-B film with the $(\mathrm{BH})_{\max }$ value of $70 \mathrm{~kJ} / \mathrm{m}^{3}$ could be obtained. After polishing a Pr-Fe-B thick-film deposited on a glass substrate with groove, the magnetic properties of the film didn't deteriorate.
\end{abstract}

Index Terms_-Pr-Fe-B film magnet, Glass substrate, PLD (Pulsed Laser Deposition), MEMS (Micro-Electro-Mechanical-Systems)

\section{INTRODUCTION}

Cilm magnets, such as a Nd-Fe-B, Sm-Co or Fe-Pt film, Fere prepared by a lot of researchers using several deposition methods [1]-[18]. Our group has focused on a PLD method to obtain isotropic Nd-Fe-B thick-film magnets with the maximum thickness up to $1250 \mu \mathrm{m}$ on metal substrates (Ta, Fe, W) under the relatively high deposition rate of several-ten-microns per hour and applied the films to several miniaturized electronic devices [19]-[21].

Although a Nd-Fe-B film thicker than $5 \mu \mathrm{m}$ on a $\mathrm{Si}$ substrate without a buffer layer was fabricated using a sputtering method, the film was peeled out from the substrate through the preparation process [22]. In order to suppress the mechanical deterioration, a Ta buffer layer between a Nd-Fe-B film and a Si substrate has been deposited and MEMS applications comprising the Nd-Fe-B films have been demonstrated using a micromachining technology [23][24]. On the other hand, the mechanical destruction of PLD-made Nd-Fe-B films on a Si substrate without a buffer layer showed a different phenomenon, namely the samples were broken from the inside of a single crystalline Si substrate (not peeling) [25]. We, therefore, prepared a $20 \mu \mathrm{m}$-thick Nd-Fe-B film magnet on a Si substrate with an approximately $1 \mu \mathrm{m}$-thick Ta buffer layer. The sample, however, was broken through a dicing process. We also confirmed that the use of the thick Ta layer was not suitable for a chemical etching process due to the ultimately low etching rate. In order to overcome the difficulty, we increased the Nd content of a PLD-made Nd-Fe$\mathrm{B}$ thick-film by taking account of the linear expansion coefficient for each material and succeeded in enhancing the thickness of the film up to $160 \mu \mathrm{m}$ on a Si substrate without a buffer layer [25].

This contribution reports the deposition of a rare-earth film magnet on a glass substrate, namely we focused on a glass MEMS [24] instead of a Si one. Furthermore, in order to enhance coercivity, we prepared isotropic PrFe-B film magnets instead of previously reported Nd-Fe-B ones because the magnetic crystalline anisotropy constant of a $\mathrm{Pr}_{2} \mathrm{Fe}_{14} \mathrm{~B}$ phase $\left(\mathrm{K}_{\mathrm{u}}=6.8 \mathrm{MJ} / \mathrm{m}^{3}\right)$ is larger by approximately $2.3 \mathrm{MJ} / \mathrm{m}^{3}$ than that of a $\mathrm{Nd}_{2} \mathrm{Fe}_{14} \mathrm{~B}$ phase $\left(\mathrm{K}_{\mathrm{u}}=4.5 \mathrm{MJ} / \mathrm{m}^{3}\right)$
[26]-[28]. In this study, an investigation on the magnetic and mechanical properties of Pr-Fe-B films deposited on glass substrates without a buffer layer was carried out. Moreover, we evaluated the magnetic properties after polishing a Pr-Fe-B thick-film magnet on a glass substrate with groove.

\section{EXPERIMENTAL}

Each film was deposited on a glass substrate (TEMPAX Float, Schott AG : $5 \mathrm{~mm} \times 5 \mathrm{~mm}$ square) using a Nd-YAG pulse laser (wave length : $355 \mathrm{~nm}$, frequency : $30 \mathrm{~Hz}$ ) in the vacuum atmosphere of approximately $10^{-5} \mathrm{~Pa}$. The laser beam was defocused on the surface of a $\operatorname{Pr}_{x} \mathrm{Fe}_{14} \mathrm{~B}(\mathrm{X}=1.8,2.0,2.2,2.4)$ target. The laser energy density was estimated at $50 \mathrm{~mJ} / \mathrm{mm}^{2}$. The distance between a target and a glass substrate was fixed at $10 \mathrm{~mm}$ in order to obtain the deposition rate of approximately $30 \mu \mathrm{m} / \mathrm{h}$.

All the as-deposited films had amorphous structure, a pulse annealing (PA) method was carried out to obtain $\mathrm{Pr}_{2} \mathrm{Fe}_{14} \mathrm{~B}$ phase [29]. The annealing time range was from 3.9 to $4.3 \mathrm{~s}$ with an infrared furnace at output power of $8 \mathrm{~kW}$, and then they were cooled down to room temperature. The estimated value of annealing temperature is considered to be higher than $823 \mathrm{~K}$ according to the previous result [30]. The magnetic properties of the samples were measured with a vibrating sample magnetometer (VSM) under the maximum applied magnetic field of $2.5 \mathrm{~T}$ after magnetizing each sample with a pulsed magnetic field of $7 \mathrm{~T}$. All the films had isotropic magnetic properties, therefore in-plane ones were only shown in the paper. The film thickness was measured with a micrometer, and the composition of each film was analyzed with an energy dispersive X-ray spectrometry (EDX). The crystalline structure of each film was observed by using a XRD (X-ray diffraction). The cross sectional view was observed by using a SEM (Scanning Electron Microscope).

\section{RESUltS AND DisCUSSION}

A. Preparation of Isotropic Pr-Fe-B Film Magnets Using Pr$\mathrm{Fe}-\mathrm{B}$ Targets with Various Compositions on Glass Substrate 
Figure 1 shows the relationship between coercivity and residual magnetic polarization of annealed isotropic Pr-Fe-B and Nd-Fe-B films deposited on glass or Ta substrates. The rare earth ( $\mathrm{Pr}$ or $\mathrm{Nd}$ ) amount of each film was controlled between 15.5 and 17.5 at. \% by using two targets of $\operatorname{Pr}_{2.4} \mathrm{Fe}_{14} \mathrm{~B}$ [11] and $\mathrm{Nd}_{2.4} \mathrm{Fe}_{14} \mathrm{~B}$ [19], respectively. The thickness range was between 20 and $40 \mu \mathrm{m}$. Use of a Pr-Fe-B target instead of a Nd-Fe-B one enabled us to increase the coercivity due to the larger crystalline anisotropy of $\mathrm{Pr}_{2} \mathrm{Fe}_{14} \mathrm{~B}$ phase compared to $\mathrm{Nd}_{2} \mathrm{Fe}_{14} \mathrm{~B}$ one [26]. Although the magnetic properties of $\mathrm{Pr}-$ Fe-B films deposited on glass substrates scattered, we could obtain similar magnetic properties of Pr-Fe-B films on both substrates. The result is considered to be attributed that the both samples were mainly composed of $\mathrm{Pr}_{2} \mathrm{Fe}_{14} \mathrm{~B}$ phase as shown in Fig. 2. In this experiment, all the films had isotropic magnetic properties and the magnetic properties such as the coercive force were inferior than that those of previouslyreported sputtering-fabricated anisotropic Nd-Fe-B [2] together with Pr-Fe-B [27][28] films. We have already prepared PLD-made anisotropic Nd-Fe-B films on metal substrates using a substrate heating system [21][31]. The preparation of anisotropic Pr-Fe-B films deposited on Si substrates is required as a future work. Furthermore, the magnetic properties of Pr-Fe-B films thicker than $20 \mu \mathrm{m}$ on glass substrates were evaluated. (see Fig. 3) Coercivity decreased and residual magnetic polarization increased with

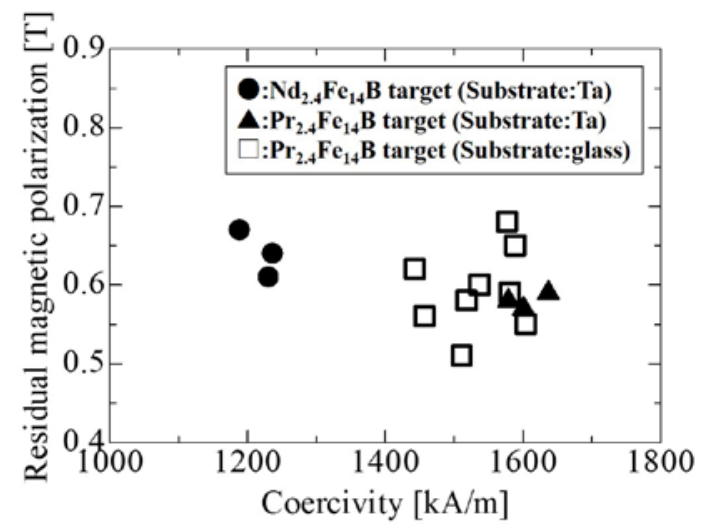

Fig. 1 Coercivity and residual magnetic polarization values of annealed Pr-Fe$\mathrm{B}$ and Nd-Fe-B thick-films deposited on each substrate. The rare earth content range of all the films was from 15.5 to 17.5 at. \%.

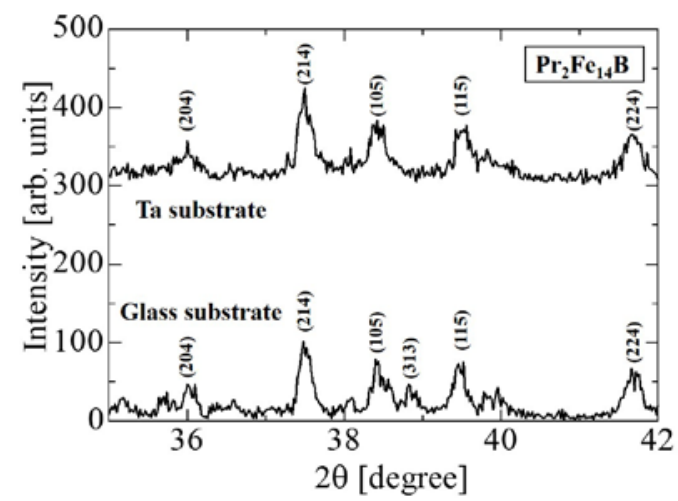

Fig. 2 X-ray diffraction patterns of Pr-Fe-B films deposited on glass and Ta substrates, respectively, using a $\mathrm{Pr}_{2.4} \mathrm{Fe}_{14} \mathrm{~B}$ target. All the diffraction peaks were corresponding to $\mathrm{Pr}_{2} \mathrm{Fe}_{14} \mathrm{~B}$ phase.

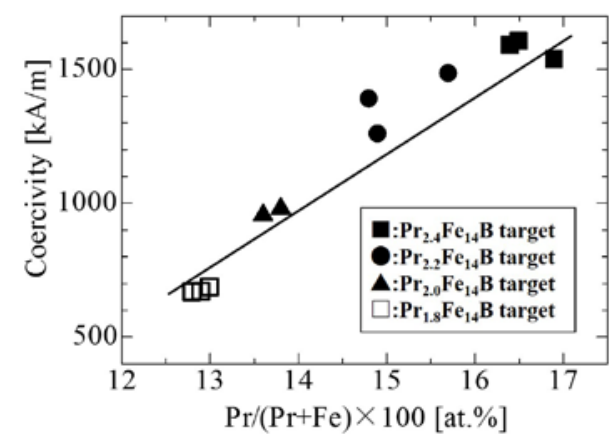

(a) Coercivity

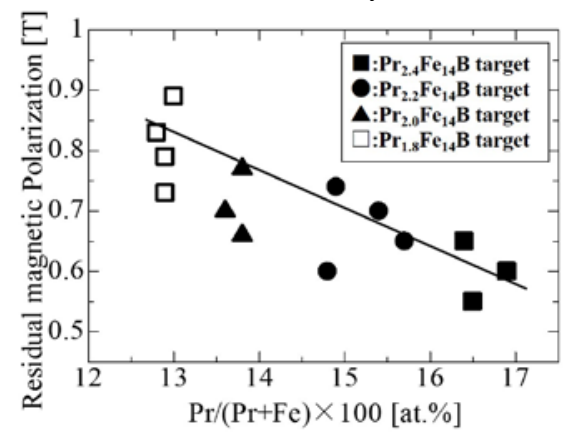

(b) Residual magnetic polarization

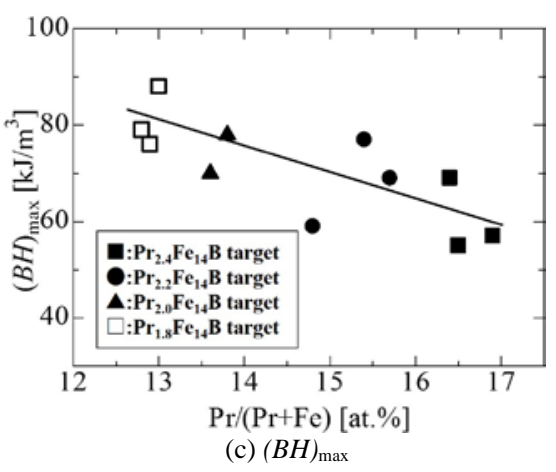

Fig. 3 Coercivity, residual magnetic polarization and $(B H)_{\max }$ values of annealed Pr-Fe-B thick-films deposited on glass substrates. Residual magnetic polarization reached up to approximately $0.8 \mathrm{~T}$ as $\mathrm{Pr} /(\mathrm{Pr}+\mathrm{Fe})$ decreased down to approximately 13 at. \%.

decreasing Pr contents of the samples. The residual magnetic polarization of approximately $0.8 \mathrm{~T}$ due to the reduction of $\mathrm{Pr}$ amount enabled us to obtain a Pr-Fe-B film with $(B H)_{\max }$ above $70 \mathrm{~kJ} / \mathrm{m}^{3}$ on a glass substrate.

B. Enhancement in thickness of isotropic Pr-Fe-B film magnets deposited on glass substrates

In order to apply Pr-Fe-B films to MEMS, the thickness of Pr-Fe-B film magnets was enhanced by increasing the deposition time as shown in Fig. 4. The value of $\mathrm{Pr} /(\mathrm{Pr}+\mathrm{Fe})$ for each film thicker than $50 \mu \mathrm{m}$ varied from 13 to 15 at. \%. It was confirmed that a mechanical destruction didn't occur in all the samples shown in Fig. 4. The phenomenon was different from the result of Nd-Fe-B films deposited on Si substrates [25]. We considered that it is attributed to the different structure of a glass (amorphous) and a Si substrate (a single crystalline structure). 


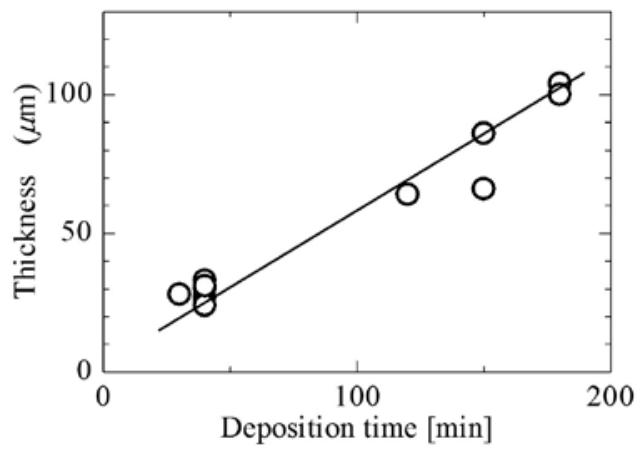

Fig. 4 Thickness of Pr-Fe-B films deposited on glass substrates as a function of deposition time. The deposition rate was estimated at about $30 \mu \mathrm{m} / \mathrm{h}$.

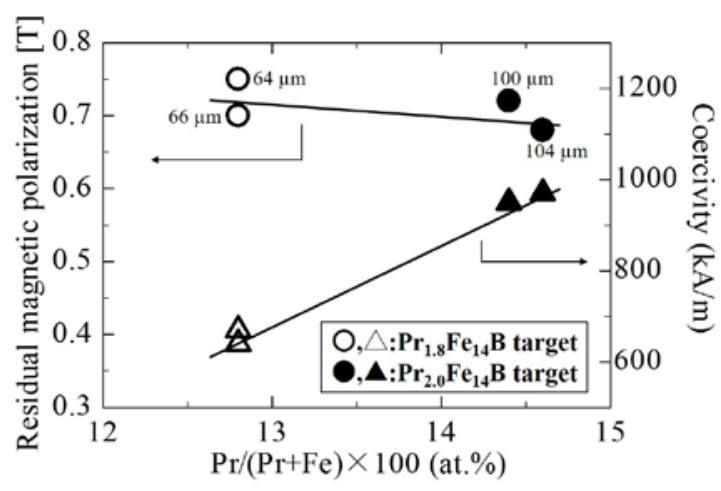

Fig. 5 Residual magnetic polarization $\left(\mathrm{J}_{\mathrm{r}}\right)$ and coercivity $\left(\mathrm{H}_{\mathrm{c}}\right)$ as a function of $\mathrm{Pr} /(\mathrm{Pr}+\mathrm{Fe})$ in Pr-Fe-B film magnets thicker than $50 \mu \mathrm{m}$ on glass substrates. $\mathrm{J}_{\mathrm{r}}$ slightly decreased and $\mathrm{H}_{\mathrm{c}}$ increased as the Pr contents increased.

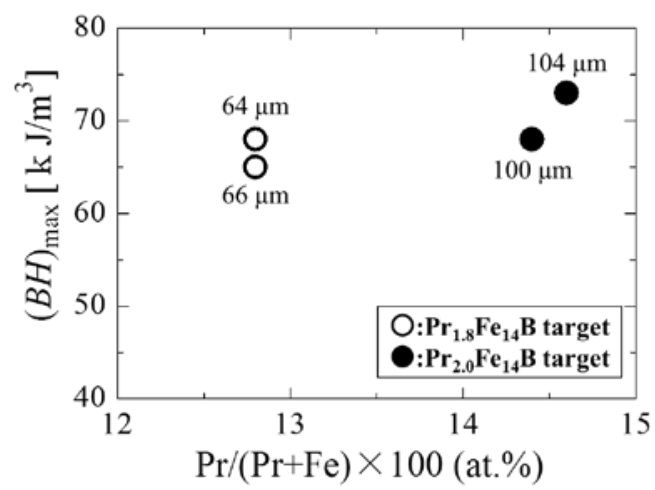

Fig. 6 Relationship between Pr contents and the $(B H)_{\max }$ values of Pr-Fe-B films displayed in Fig. 5. (BH $)_{\max }$ values of all the samples exceeded $60 \mathrm{~kJ} / \mathrm{m}^{3}$.

Magnetic properties of Pr-Fe-B films thicker than $50 \mu \mathrm{m}$ on glass substrates were displayed in Figs. 5 and 6. Increase in coercivity and slightly decrease in residual polarization occurred with increasing $\operatorname{Pr}$ contents. The $(B H)_{\max }$ range of all the samples exceeded $60 \mathrm{~kJ} / \mathrm{m}^{3}$. Figure 7 shows a J-H loop of an approximately $100 \mu \mathrm{m}$-thick Pr-Fe-B film. An observation on crystalline structure using an XRD revealed that the obtained hard magnetic properties is attributed to the formation of $\mathrm{Pr}_{2} \mathrm{Fe}_{14} \mathrm{~B}$ phase.

\section{A $100 \mu$ m-thick Pr-Fe-B thick-film magnet deposited on glass mold substrates}

Figure 8 (a) shows a Pr-Fe-B thick film magnet with the thickness of approximately $100 \mu \mathrm{m}$ on a glass substrate with groove. Several vacancies due to the existence of large

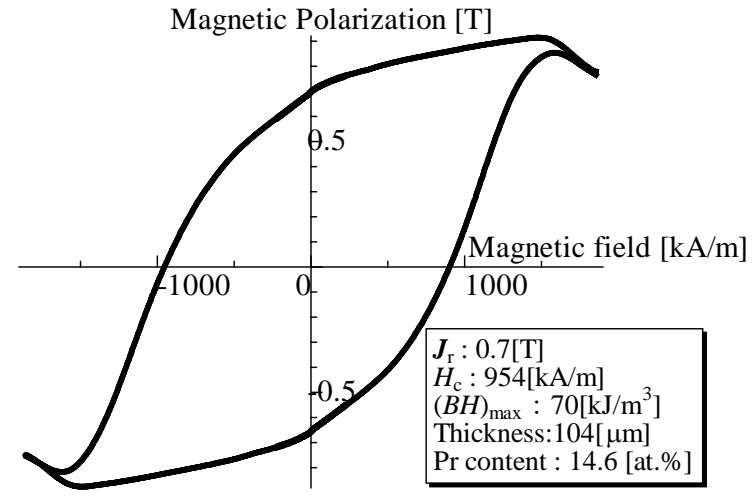

Fig. 7 A J-H loop of an approximately $100 \mu$ m-thick Pr-Fe-B film magnet with the $(B H)_{\max }$ value of $70 \mathrm{~kJ} / \mathrm{m}^{3}$.

aggregates emitted from the targets during the deposition could be observed. The depth and width of the grooves were 50 and $100 \mu \mathrm{m}$, respectively. As shown in Fig. 8 (b), the sample was polished in order to obtain the flat surface. The magnetic properties of each sample displayed in Figs. 8 (a) and (b) didn't deteriorate after the polishing. (see Fig. 9) We confirmed that the effect of above-mentioned large aggregates was little in the polishing process. Namely, it was clarified that the PLD method is effective to obtain a thick film magnet applicable to a glass MEMS.

\section{ACKNOWLEDGMENT}

This work was supported in part by JSPS KAKENHI Grant Number JP 15H03978.

\section{SUMMARY}

In the study, isotropic Pr-Fe-B thick-film magnets could be prepared on glass substrates under the high deposition rate of $30 \mu \mathrm{m} / \mathrm{h}$. The obtained results are as follows;

1. The obtained maximum thickness reached approximately $100 \mu \mathrm{m}$ without mechanical destruction.

2. The $(B H)_{\max }$ values of all the annealed Pr-Fe-B samples thicker than $50 \mu \mathrm{m}$ exceeded $60 \mathrm{~kJ} / \mathrm{m}^{3}$.

3. A Pr-Fe-B thick-film magnet prepared using large aggregates could be deposited on a glass substrate with groove. After polishing the sample, the magnetic properties didn't deteriorate.

\section{REFERENCES}

[1] F. Dumas-Bouchat, L. F. Zanini, M. Kustov, N. M. Dempsey, R. Grechishkin, K. Hasselbach, J. C. Orlianges, C. Champeaux, A. Catherinot, and D. Givord, Appl. Phys. Lett. , "Thermomagnetically patterned micromagnets”, vol. 96, 102511, 2010.

[2] N. M. Dempsey, A. Walther, F. May, D. Givord, K. Khlopkov, and O. Gutfleisch, Appl. Phys. Lett. " High performance hard magnetic NdFeB thick films for integration into micro-electro-mechanical systems”, vol. 90, 092509, 2007.

[3] M. Uehara, J. Magn. Magn. Mater. ,’Microstructure and permanent magnet properties of a perpendicular anisotropic $\mathrm{NdFeB} / \mathrm{Ta}$ multilayered thin film prepared by magnetron sputtering”, vol. 284, pp. 281-286, 2004. 


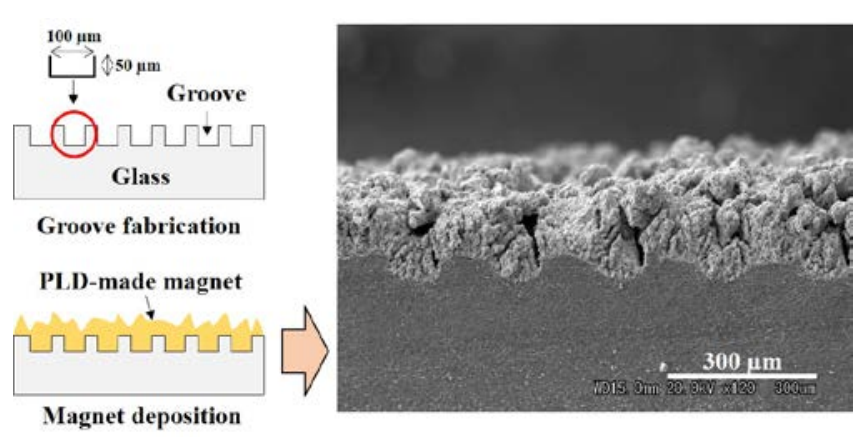

(a) After deposition

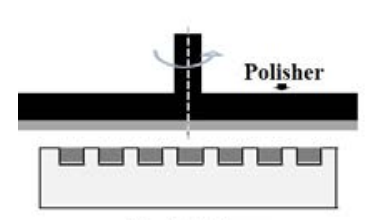

Polishing

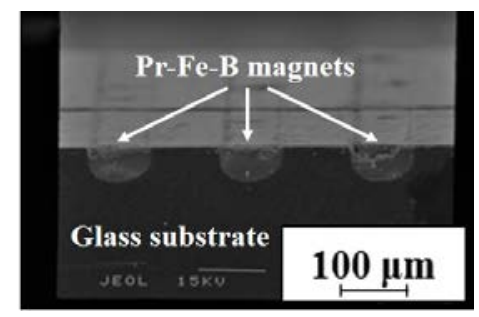

(b) After polishing

Fig. 8 Cross sectional view of a Pr-Fe-B thick-film magnets deposited on a substrate with groove (a) and the polished film (b).

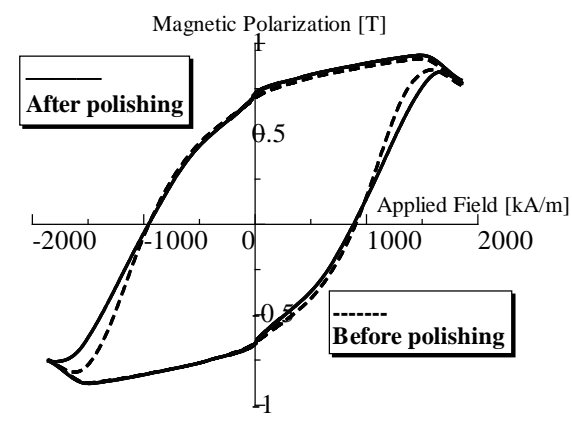

Fig. $9 \mathrm{~J}-\mathrm{H}$ loop of a polished Pr-Fe-B film magnet with the thickness of approximately $100 \mu \mathrm{m}$ on a glass substrate with groove. In order to compare the magnetic properties, a loop of the sample before polishing was also shown.

[4] L. K. B. Serrona, A. Sugimura, N. Adachi, T. Okuda, H. Ohsato, I. Sakamoto, A. Nakanishi, M. Motokawa, D. H. Ping, and K. Hono, Appl. Phys. Lett. "'Structure and magnetic properties of high coercive NdFeB films with a perpendicular anisotropy", vol. 82, 1751, 2003.

[5] B. A. Kapitanov, N. V. Kornilov, Ya. L. Linetsky, and V. Yu. Tsvetkov, J. Magn. Magn. Mater. ,"Sputtered permanent Nd-Fe-B magnets”, vol. 127, pp. 289-7, 1993.

[6] F. J. Cadieu, H. Hegde, E. Schloemann, and H. J. Van Hook, J. Appl. Phys. , "In-plane magnetized YIG substrates self - biased by SmCo based sputtered film coatings“, vol. 76, 6059, 1994.

[7] F. J. Cadieu, R. Rani, X. R. Qian, and L. Chen, J. Appl. Phys. , ”High coercivity SmCo based films made by pulsed laser deposition“", vol. 83, $6247,1998$.

[8] F. J. Cadieu, R. Rani, T. Theodoropoulos, and Li Chen, J. Appl. Phys. , "Fully in plane aligned SmCo based films prepared by pulsed laser deposition“, vol. 85, 5895, 1999.

[9] H. Aoyama and Y. Honkura, J. Magn. Soc. Jpn.,"'Magnetic Properties of Fe-Pt Sputtered Thick Film Magnet“, vol. 20, No.2 pp. 237-240 , 1996. [in Japanese]

[10] M. Nakano, H. Kuroda, J. Koga, T. Yanai, Y. Tanaka, and H. Fukunaga, J. Appl. Phys. , "Order/disorder phase composite Fe-Pt exchange-spring thick film magnets“, vol. 103, pp. 07E1101-07E1103 , 2008.
[11] M. Nakano, W. Oniki, T. Yanai, and H. Fukunaga, J. Appl. Phys. , "Magnetic properties of pulsed laser deposition-fabricated isotropic Fe-Pt film magnets“, vol. 109, pp. 07A7231-07A7234 , 2011.

[12] W. F. Liu, S. Suzuki, D. S. Li, and K. Machida, J. Magn. Magn. Mater. ,"Magnetic properties of Fe-Pt thick-film magnets prepared by RF sputtering“', vol. 302, pp. 201-205, 2006.

[13] S. Yamashita, J. Yamasaki, M. Ikeda, and N. Iwabuchi, J. Appl. Phys. , "Anisotropic Nd-Fe-B thin - film magnets for milli-size motor", vol. 70, 6627, 1991.

[14] O. Cugat, Proc. 16th Int. Workshop Rare Earth Magnets and Their Applications, " RARE EARTH PERMANENT MAGNET APPLICATIONS IN MAGMAS “, p. 478, 2002.

[15] P. McGuiness, D. Jezersek, S. Kobe, B. Markoh, S. Spaic, and B. Saje, J. Magn. Magn. Mater. "100- $\mu$ m-thick Nd-Fe-B magnets for MEMS applications produced via a low-temperature sintering route“, vol. 305, 177-181, 2006.

[16] A. Walther, C. Marcoux, B. Desloges, R. Grechishkin, D. Givord, and N. M. Dempsey, J. Magn. Magn. Mater. ,"Micro-patterning of NdFeB and SmCo magnet films for integration into micro-electro-mechanicalsystems“, vol. 321, pp. 590-594, 2009.

[17] R. Fujiwara, T. Shinshi, and M. Uehara, Int. J. Automat. Technol. , "Positioning Characteristics of a MEMS Linear Motor Utilizing a Thin Film Permanent Magnet and DLC Coating“, vol. 7, pp. $148-155,2013$.

[18] T. Budde and H. Gatzen, J. Appl. Phys.,’Thin film SmCo magnets for use in electromagnetic microactuators“, vol. 99, 08N304, 2006.

[19] M. Nakano, H. Takeda, F. Yamashita, T. Yanai, and H. Fukunaga, IEEE Trans. Magn. ,'Improvement in Magnetic Properties of PLD-Made NdFe-B Thick Film Magnets“, vol. 44, pp. 4199-4202, 2008.

[20] M. Nakano, K. Motomura, T. Yanai, and H. Fukunaga, IEEE Trans. on Magn. , "Nanocomposite Thick-Film Magnets with Nd-Fe-B $+\alpha$-Fe Phases Prepared under High Laser Energy Density“, vol. 50, pp. 21014041-21014044, 2014.

[21] M. Nakano, S. Sato, F. Yamashita, T. Honda, J. Yamasaki, K. Ishiyama, M. Itakura, J. Fidler, T. Yanai, and H. Fukunaga, IEEE Trans. Magn. "Review of Fabrication and Characterization of Nd-Fe-B Thick Films for Magnetic Micromachines“, vol. 43, pp. 2672-2676, 2007.

[22] Y. Zhang, D. Givord, and N. M. Dempsey, Acta Materialia,’The influence of buffer/capping-layer-mediated stress on the coercivity of NdFeB films”, vol. 60, pp. 3783-3788, 2012.

[23] C. Zhi, T. Shinshi, and M. Uehara, International, J. Automation Technplogy,"Design and Analysis of a Thin Film Permanent Magnet Actuated Micro Pump“, vol. 7, pp. 196-204, 2013.

[24] R. Fujiwara, T. Shinshi, E. Kazawa, Sensors and Actuators A, "Micromagnetizaton patterning of sputtered NdFeB/Ta multilayered films utilizing laser assisted heating”, vol. 220, pp. 298-304, 2014.

[25] M. Nakano, Y. Chikuba, M. Oryoshi, A. Yamashita, T. Yanai, R. Fujiwara, T. Shinshi, H. Fukunaga, IEEE Trans. Magn. , "Nd-Fe-B film magnets with thickness above 100 um deposited on Si substrates", vol. 51, p. 7156598, 2015.

[26] K. H. J. Buschow in Ferromagnetic Materials (North -Holland Publishing), 4, 1988.

[27] F. Yang, W. Liu, W. B. Ciu, J. N. Feng, Y. Q. Zhang, Z. D. Zhang, Physica B, "Thickness dependence of the magnetic properties of highcoercive Pr-Fe-B thin films with perpendicular magnetic anisotropy”, vol. 403, pp. 3631-3634, 2008.

[28] F. Yang, W. Liu, W. B. Ciu, Q. Yao, X. G. Zhao, Z. D. Zhang, Materials Letters, "Structure and magnetic properties of high-coercive $\left[\mathrm{PrFeB}_{\mathrm{X}} / \mathrm{Cu}\right]_{\mathrm{n}}$ films with out-of-plane orientation”, vol. 63, pp. 18661868, 2009.

[29] H. Fukunaga, K. Tokunaga, and J. M. Song, IEEE Trans. Magn. , "Improvement in coercivity by high-speed crystallization for PrFeB based nanocomposite magnets”, vol. 38, pp. 2970-2972, 2002.

[30] M. Nakano, S. Tutumi, H. Fukunaga and J. M. Song, IEEE Trans. Magn. , "Preparation of Nd-Fe-B thin film magnets with high coercivity using laser ablation technique”, 37, pp. 2573-2575, 2001.

[31] M. Nakano, S. Sato, H. Fukunaga, and F. Yamashita, J. Appl. Phys. , ”A method of preparing anisotropic Nd-Fe-B film magnets by pulsed laser deposition“, vol. 99, 08N301 , 2006. 